\title{
Intractable Scapular Pain Due to Undiagnosed Osteoid Osteoma: A Case Report
}

\author{
Karthika Veerapaneni ${ }^{1}$ Poornachand Veerapaneni ${ }^{1}$ Nidhi Kapoor ${ }^{1}$ Rohan S Samant ${ }^{2}$ \\ Sisira Yadala ${ }^{1}$ Sen Sheng ${ }^{1}$ Krishna Nalleballe ${ }^{1, \odot}$
}

${ }^{1}$ Department of Neurology, University of Arkansas for Medical Sciences, Little Rock, Arkansas, United States

${ }^{2}$ Department of Radiology, University of Arkansas for Medical

Sciences, Little Rock, Arkansas, United States

\begin{abstract}
Address for correspondence Karthika Veerapaneni, MD, Department of Neurology, University of Arkansas for Medical Sciences, Little Rock, AR 72205, United States (e-mail: kveerapaneni@uams.edu).
\end{abstract}

J Neurosci Rural Pract:2020;11:489-491

\begin{abstract}
A 36-year-old female patient presented to our stroke neurology clinic for progressively worsening intractable, sharp, shooting interscapular pain radiating to the right shoulder and neck, which she had experienced for 4 years. She had previously seen an orthopedist and was referred to a neurosurgeon for surgical intervention after an MRI of the cervical spine showed the C3-C4 right vertebral artery loop protruding into the right C3-C4 neural foramen and compressing the exiting C4 nerve root. MR neurography showed a stable tortuous right vertebral artery loop, causing a mass effect on the dorsal root ganglion. A neuroforaminal decompression surgery was planned. However,

Keywords

- magnetic resonance imaging

- neuro-oncology

- neuroradiology

- osteoid osteoma

- pain

- spinal cord the patient visited our stroke neurology clinic for a second opinion before surgery. An MRI of the thoracic spine showed an enhancing soft tissue mass at the right T4-T5 pedicles and adjacent body. A chest CT with contrast showed a $1 \mathrm{~cm}$ radiolucent lesion in the superior articular facet of T5, which represented a nidus. A technetium bone scan showed focal increased uptake within the right T5 pedicle, which is indicative of osteoid osteoma. The patient underwent laminectomy/resection and was pain-free at a 6-month follow-up; biopsy confirmed osteoid osteoma. This case illustrates the importance of neurolocalization during diagnostic testing.
\end{abstract}

\section{Introduction}

Osteoid osteoma is a rare, benign bone-forming tumor first described in 1935 by Jaffe. ${ }^{1}$ These tumors usually occur in people between 10 and 35 years of age and are more common in males. Osteoid osteomas are typically found in the long bones, but they also occur in the spine. The most common symptom is pain, but patients with spine lesions may present with a limp or neurologic symptoms secondary to spinal cord or nerve root compression. ${ }^{2}$ CT is the modality of choice to evaluate osteoid osteomas, which typically show a lucent nidus with surrounding sclerotic reactive bone. MR neurography is the best modality to determine the effects of the tumor on the spinal cord, and a technetium bone scan can accurately localize the tumor and differentiate it from osteomyelitis. ${ }^{3}$ Treatment for osteoid osteoma generally involves the total resection of the nidus. Percutaneous radiofrequency thermal ablation ${ }^{4}$ and laser photocoagulation ${ }^{5}$ have recently been promoted as less invasive options, but they should be used with caution in spinal lesions because of the risk of neuronal damage and a higher rate of recurrence. ${ }^{4,5}$ The prognosis is good if the lesion is removed completely.

\section{Case Presentation}

A 36-year-old female presented to our stroke neurology clinic for interscapular pain that had worsened progressively over 4 years. The pain was sharp, severe, and radiated to the right shoulder, right anterior chest, and right side of the neck. During these 4 years, the patient was not able to perform her job or take care of her child due to the pain. She had also been seen by multiple physicians in and outside of the 
United States before ultimately being diagnosed with osteoid osteoma.

The patient was initially evaluated at an orthopedic clinic. An MRI of the right shoulder was unrevealing, but she was referred to a neurosurgeon for surgical intervention after an MRI of the cervical spine showed the C3-C4 right vertebral artery loop protruding into the right $\mathrm{C} 3-\mathrm{C} 4$ neural foramen and compressing the exiting C4 nerve root. The neurosurgeon evaluated her and ordered MR neurography, which showed a stable tortuous right vertebral loop, causing a mild mass effect on the dorsal root ganglion. Neuroforaminal decompression was recommended.

She then came to our stroke neurology clinic for a second opinion before surgery. A neurological examination found mildly impaired finger strength in her right hand, but no other deficits. An MRI of the thoracic spine with contrast was performed because of the interscapular pain; the MRI showed an ill-defined enhancing soft tissue mass with contrast uptake at the right T4-T5 pillars, pedicles, and adjacent body, and encasement of the exiting right $\mathrm{T} 4$ and $\mathrm{T} 5$ nerve roots. (-Fig. 1). Hypointensity at $\mathrm{T} 1$ and $\mathrm{T} 2$ was consistent with sclerotic changes. With infectious etiology as a differential diagnosis, a chest CT with contrast was performed, which showed sclerosis of the right T4-T5 articular pillars and a $1 \mathrm{~cm}$ radiolucent lesion in the superior articular facet of T5, which represented a nidus (-Fig. 2). A technetium bone scan

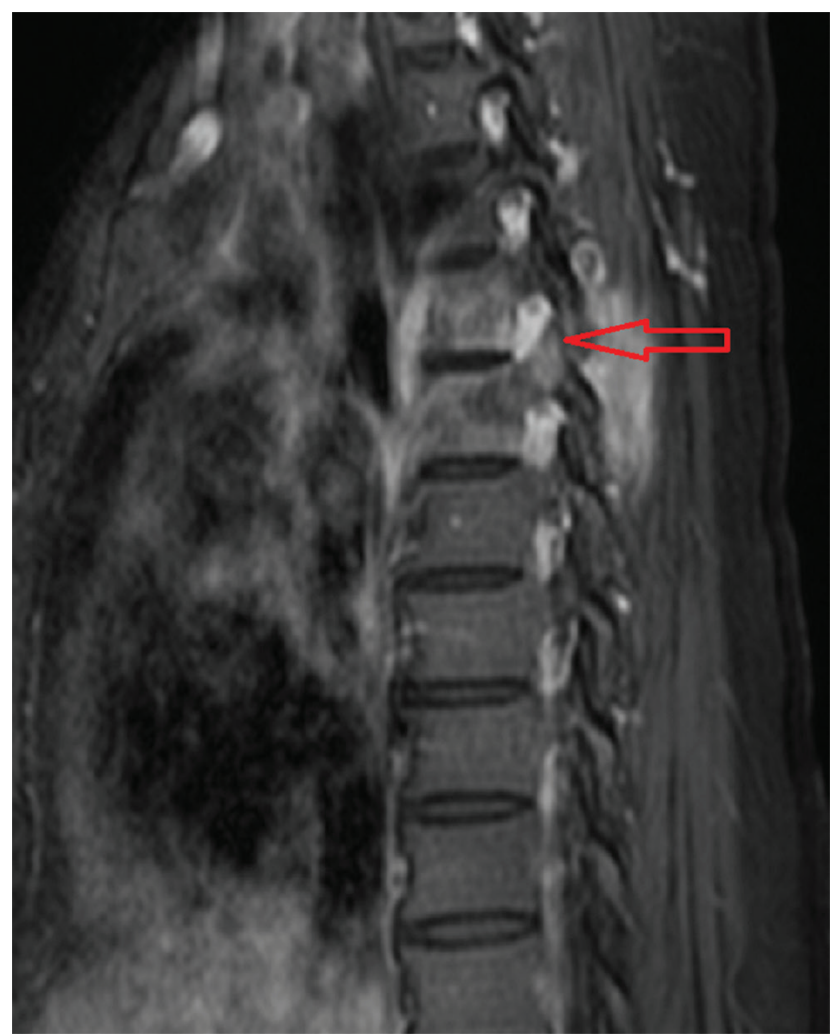

Fig. 1 MRI showing an ill-defined enhanced soft tissue mass with contrast uptake at the right T4-T5 pillars, pedicles, and adjacent body, with encasement of the exiting right T4 and T5 nerve roots. MRI, magnetic resonance imaging. showed focal increased uptake within the right $\mathrm{T} 5$ pedicle, which is indicative of osteoid osteoma. A biopsy could not be performed due to the location. The patient underwent hemilaminectomy through a posterior approach, with resection of inferior articulating process of $\mathrm{T} 4$ and superior articulating process of $\mathrm{T} 5$ for tumor resection along with arthrodesis of right T4-T5 as well as allograft and microsurgical technique for resection of tumor mass. Pathology showed findings of osteoblastoma (-Figs. 3 and - Fig. 4 ). She was re-evaluated 9 months after surgery and remained pain-free with no residual neurological deficits.

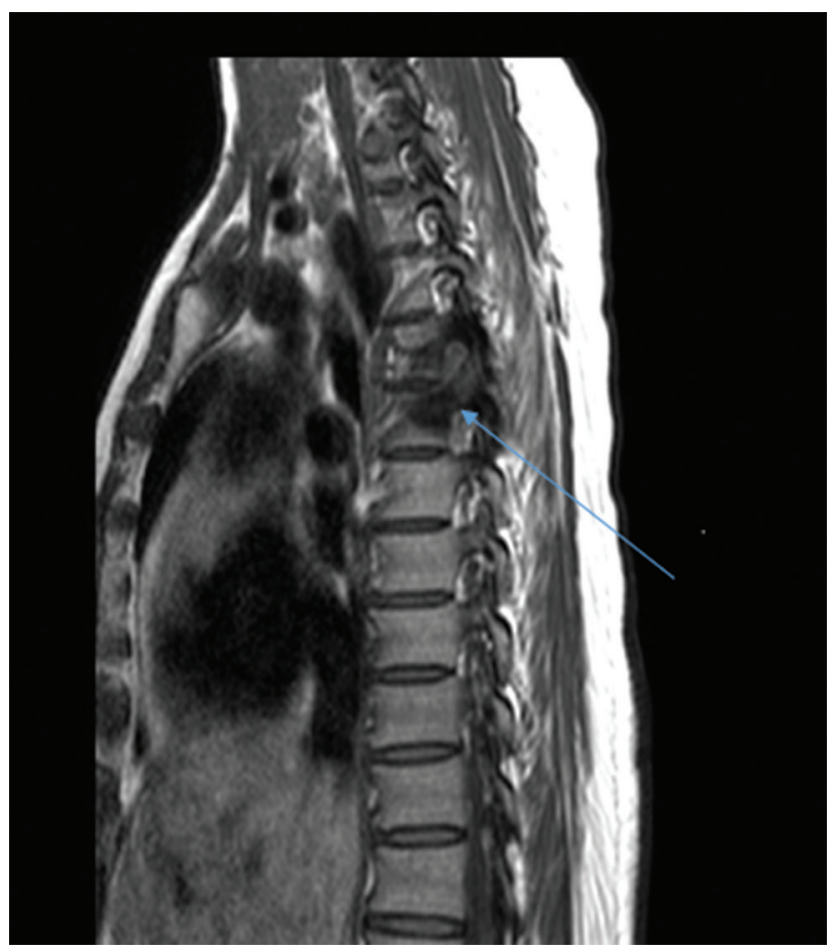

Fig. 2 CT with contrast showing sclerosis of the right T4-T5 articular pillars and a $1 \mathrm{~cm}$ radiolucent lesion in the superior articular facet of T5, which represented a nidus. CT, computerized tomography.

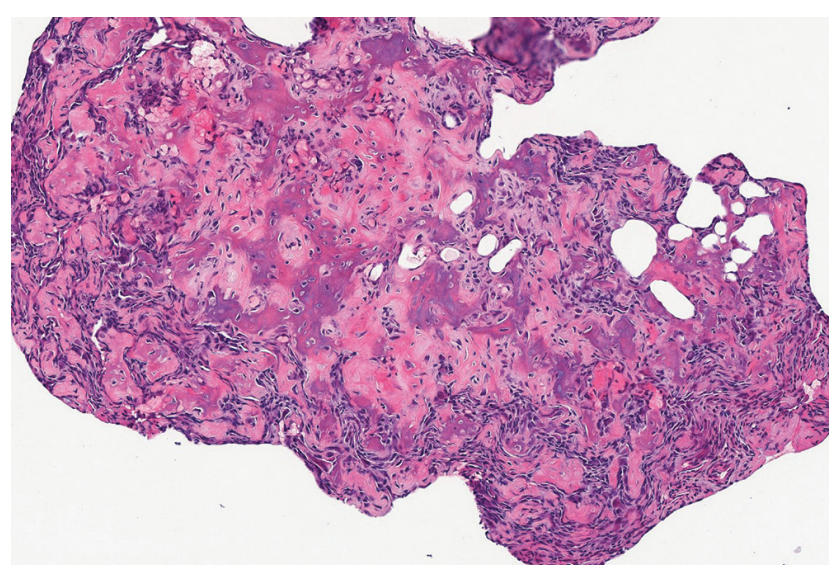

Fig. 3 The normal bone has been replaced by disorganized new woven bone. Irregular pink to purple strands of osteoid are arranged haphazardly with intervening osteoblasts (hematoxylin and eosin stain; 120x magnification; image courtesy of Jerad M. Gardner, MD). 


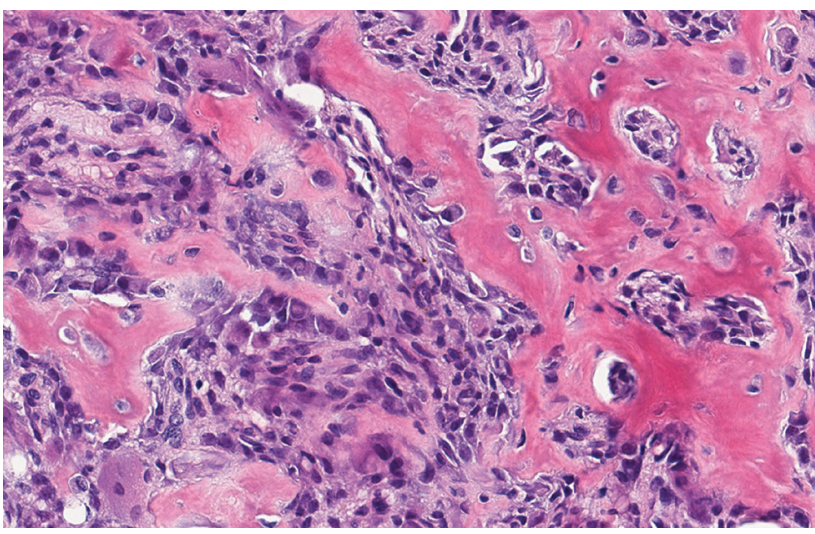

Fig. 4 Plump purple osteoblasts are depositing strands of pink osteoid matrix (hematoxylin and eosin stain; 360x magnification; image courtesy of Jerad M. Gardner, MD).

\section{Discussion}

This patient started experiencing interscapular pain 4 years before coming to our stroke clinic. Because the interscapular pain radiated to the right shoulder and neck, she was initially referred to an orthopedist and neurosurgeon. She underwent an MRI of the right shoulder, which was unremarkable. However, an MRI of the cervical spine showed the right vertebral artery loop compressing the C4 nerve root; even though neurography showed a mild stable mass effect and her pain was progressively worsening, this was believed to be the cause of her pain. Once this diagnosis was established, all of the physicians that she saw subsequently, here and abroad, assumed that her pain was caused by the vertebral artery loop, even though her pain was primarily in the thoracic area, and no imaging of the thoracic spine was performed.

In this era of imaging, we still strongly believe that the neurological examination plays a crucial role in localization, as illustrated by this case. Another important point is premature closure, which is defined as the failure to consider alternative diagnoses after an initial impression is formed. Premature closure seems to have played a key role in this case, as the patient had experienced this intractable pain for 4 years. Once the diagnosis of a vertebral artery loop was made, multiple physicians all attributed her pain to this diagnosis. This patient could have been treated sooner if her physicians had followed good medical practice guidelines by adequately assessing patient with a good history and physical examination with broad differential diagnoses or referred patient for second opinion or consulted a colleague.

\section{Conclusion}

This case illustrates the importance of following good medical practice guidelines such as adequate assessment of patient condition with good history and neurological examination in localizing spinal cord lesions and having broad differential diagnoses to prevent premature closure along with the importance of second opinion when in doubt.

\section{Funding \\ None. \\ Conflict of Interest \\ None declared.}

\section{References}

1 Jaffe HL. Benign osteoblastoma. Bull Hosp Jt Dis 1956;17(2): 141-151

2 Azouz EM, Kozlowski K, Marton D, Sprague P, Zerhouni A, Asselah F. Osteoid osteoma and osteoblastoma of the spine in children. Report of 22 cases with brief literature review. Pediatr Radiol 1986;16(1):25-31

3 Pettine KA, Klassen RA. Osteoid-osteoma and osteoblastoma of the spine. J Bone Joint Surg Am 1986;68(3):354-361

4 Pinto $\mathrm{CH}$, Taminiau AH, Vanderschueren GM, Hogendoorn PC, Bloem JL, Obermann WR. Technical considerations in CT-guided radiofrequency thermal ablation of osteoid osteoma: tricks of the trade. AJR Am J Roentgenol 2002;179(6):1633-1642

5 Rosenthal DI, Hornicek FJ, Wolfe MW, Jennings LC, Gebhardt MC, Mankin HJ. Percutaneous radiofrequency coagulation of osteoid osteoma compared with operative treatment. J Bone Joint Surg Am 1998;80(6):815-821 